\title{
Relationship between GH/IGF-1 Axis, Graft Recovery, and Early Survival in Patients Undergoing Liver Transplantation
}

\author{
Angela Salso, ${ }^{1}$ Giuseppe Tisone, ${ }^{2}$ Laura Tariciotti, ${ }^{2}$ Ilaria Lenci, ${ }^{1}$ \\ Tommaso Maria Manzia, ${ }^{2}$ and Leonardo Baiocchi ${ }^{1}$ \\ ${ }^{1}$ Hepatology Unit, Department of Internal Medicine, University of Rome “Tor Vergata”, Via Montpellier 1, 00133 Rome, Italy \\ ${ }^{2}$ Transplant Unit, Department of Surgery, “Tor Vergata” University, Via Montpellier 1, 00133 Rome, Italy \\ Correspondence should be addressed to Leonardo Baiocchi; baiocchi@uniroma2.it
}

Received 10 February 2014; Revised 27 February 2014; Accepted 5 March 2014; Published 1 April 2014

Academic Editor: Salvador Benlloch

Copyright (C) 2014 Angela Salso et al. This is an open access article distributed under the Creative Commons Attribution License, which permits unrestricted use, distribution, and reproduction in any medium, provided the original work is properly cited.

Background. High levels of IGF-1 have been reported in patients with initial poor function of the graft after liver transplantation (LT). Correlation with other clinical variables or early survival has not been extensively investigated. Aim. To evaluate the GH/IGF1 profile as a function of liver recovery and patients' early survival after LT. Methods. 30 transplanted patients (23 survivors and 7 nonsurvivors), were retrospectively enrolled in the study. GH and IGF-1 serum levels were assessed at baseline, graft reperfusion, and 1, 7, 15, 30,90 , and 360 days after LT. Individual biochemical variables were also recorded. Results. After grafting, IGF-1 in blood linearly correlated with cholesterol $(r=0.6, P=0.001)$. IGF-1 levels from day 15 after surgery were statistically higher in survivors as compared to nonsurvivors. ROC curves analysis identified an IGF- 1 cut-off $>90 \mu \mathrm{g} / \mathrm{L}$, from day 15 after surgery, as a good predictor of survival (sensitivity $86 \%$, specificity 95\%, and $P<0.001$ ). Conclusions. After LT, GH levels correlate with the extent of cytolysis, while IGF-1 is an indicator of liver synthetic function recovery. IGF-1 levels $>90 \mu \mathrm{g} / \mathrm{L}$ (day 15-30) seem to be an indicator of short-term survival.

\section{Introduction}

The growth hormone/insulin like growth factor-1 (GH/IGF-1) axis is of paramount importance in growth and development and is also a lifespan determinant in animal models [1-5]. IGF-1 is considered the principal mediator of GH effects in tissues. Its production is stimulated primarily in the liver through STAT 5 pathway $[1,2]$. While the GH remains the major regulator of IGF-1 liver production, in turn IGF1 counteracts GH synthesis in a classic negative feedback system. Liver diseases such as cirrhosis are characterized by a deranged GH-IGF-1 system, with increased levels of GH and reduction in IGF-1 production [6-9]. As a result patients with liver cirrhosis exhibit GH serum levels, and half-lives, that are twofold those of normal subjects. This is possibly due to reduced GH clearance and/or acquired resistance for decreased IGF-1 liver production. Reduced IGF-1 serum levels are associated with an unfavourable prognosis in cirrhotic patients $[10,11]$. The reasons may be related to consequent malnutrition, insulin resistance, and immunologic impairment. In this perspective some attempts with replacement hormonal therapy have been made in liver cirrhosis showing encouraging results $[12,13]$. Few studies are available on the GH-IGF-1 axis in adult patients undergoing liver transplantation (LT) [14-17]. All studies show the prompt return to normal of the GF-IGF-1 axis after transplant. One of these studies attempted to analyze the GH-IGF-1 axis trend as a function of graft recovery [18]. The authors described higher levels of IGF-1 on the first day after LT in patients with Initial Poor Function (IPF) and a normalization of IGF-1 serum levels over a longer period ( $\approx 3$ months) in this category of subjects. Unfortunately, this interesting work, probably given the small number of patients, did not find correlations between fluctuation of GH-IGF-1 axis and other biochemical variables, and patient survival was not examined. In the present study we reassessed this issue, using a larger retrospective cohort of patients and including data on GH and IGF-1 in correlation with common biochemical variables and patient survival. 
TABLE 1: Baseline characteristics of patients included in the study.

\begin{tabular}{lc}
\hline Number of patients & 30 \\
Age (years) & $55.2 \pm 11.7$ \\
Gender (M/F) & $23 / 7$ \\
MELD score at transplant & $16.2 \pm 1.8$ \\
Donor age (years) & $48.7 \pm 24.8$ \\
Donor gender (M/F) & $21 / 9$ \\
Cold ischemia time (minutes) & $396 \pm 112$ \\
Warm ischemia time (minutes) & $42 \pm 16$ \\
& $12 \mathrm{HCV}$ \\
& $6 \mathrm{HBV}$ \\
Cause of liver disease & 5 Alcoholic \\
& 2 Primary sclerosing cholangitis \\
& 2 Autoimmune \\
Immunosuppression & 2 Cryptogenic \\
(cyclosporine-A/tacrolimus) & 1 Polycystic liver disease \\
\hline
\end{tabular}

Continuous variables are expressed as mean \pm standard deviation.

\section{Patients and Methods}

2.1. Patients. Thirty patients (23/7, M/F; mean age $55.2 \pm$ 11.7) undergoing LT for various indications at our institution were retrospectively selected for this study. Exclusion criteria were (i) retransplantation; (ii) multiorgan transplantation; (iii) transplantation for fulminant hepatic failure; (iv) hepatic cirrhosis complicated by hepatocarcinoma (HCC); (v) body mass index (BMI) > 30; (vi) serum samples not available for GH and IGF-1 assessment. The protocol of the retrospective study was submitted and approved by our local ethics committee. The operation was performed according to standard technique, with the use of venovenous bypass and the positioning of a T-tube to protect the biliobiliary anastomosis. The T-tube was removed three months after grafting, in compliance with the standard protocol of our and other liver units $[19,20]$. Demographic baseline features including the etiology of liver disease are included in Table 1. All patients underwent immunosuppression with either tacrolimus or cyclosporine monotherapy, and, according to our centre's protocol, no steroids or other immunosuppressants were used [21]. Ten age-matched healthy volunteers with normal liver enzymes and synthesis served as controls.

2.2. Serum Sampling. Blood samples were collected from each patient at listing, during LT (graft reperfusion phase), and on postoperative days $1,7,15,30,90$, and 360. Plasma GH and IGF-1 levels were measured using commercially available ELISA kits (Pantec, Milan, Italy) and following the vendor's instructions. Other common biochemical parameters were assessed in the central laboratory facilities of our hospital (Policlinico di Tor Vergata, University of Rome "Tor Vergata"; Rome, Italy), daily during the first month and weekly or monthly thereafter.

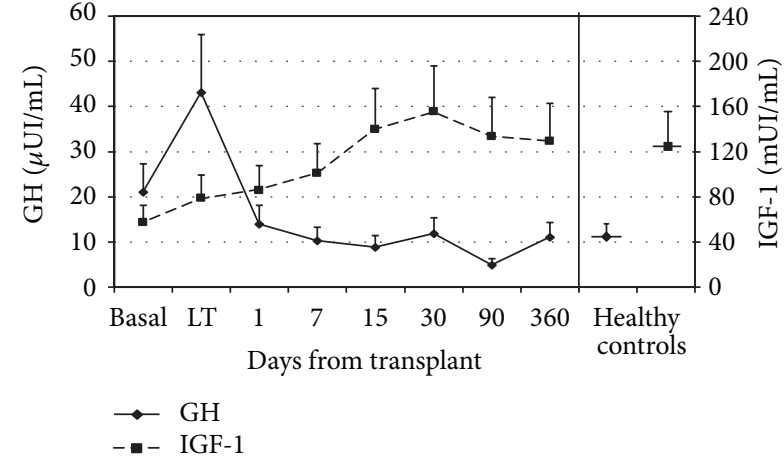

FIGURE 1: Mean values of GH and IGF-1 in the pretransplant (basal) phase, at liver transplantation (LT), and after transplant until day 360. GH and IGF-1 results are plotted according to left and right axis, respectively. Results of healthy controls (right portion of the graph) are also reported for comparison.

2.3. Statistical Analysis. The statistical analysis was performed using the Student's $t$-test for paired or unpaired data and Pearson $r$ for correlation analysis. Receiver operating characteristic (ROC) curves were designed to assess the sensitivity and the specificity of GH and IGF-1 serum levels in predicting survival, as previously described by our group in another study [22]. In brief, after ROC curves were constructed, the corresponding areas under the curves (AUC) were calculated. Next, the sensitivity, specificity, positive predictive value, and negative predictive value were calculated. The optimal cut-off value to predict mortality was determined using the Youden index method, which defines the cut-off in terms of the maximal sum of sensitivity and specificity. Survival analysis was conducted using the Kaplan Meier test and statistical differences were evaluated using the logrank test as previously described [23]. All statistical analyses were conducted using the Statistical Software Package NCSS (Kaysville, UT). A $P<0.05$ was considered statistically significant.

\section{Results}

3.1. Patients. Baseline characteristics of patients selected for the study are summarized in Table 1 . The main reason for liver transplantation was virus related ( $\mathrm{HCV}$ or $\mathrm{HBV}$ ) end stage liver disease. All patients received a whole cadaveric organ. No acute rejection episode was recorded. Seven patients died within three months of liver transplantation. In all these patients the final cause of death was multiorgan failure, originating from graft dysfunction in four, graft dysfunction associated with renal insufficiency in two, liver and renal dysfunction associated with biliary tract complications in one.

3.2. Serum Levels of GH and IGF-1. Mean levels of serum $\mathrm{GH}$ and IGF-1 at time of transplant and thereafter are shown in Figure 1 together with values of control individuals. Basal levels of GH and IGF-1 were statistically different from the control group: $\mathrm{GH} \mu \mathrm{UI} / \mathrm{mL} 21 \pm 7$ versus $12 \pm 4$ in controls 


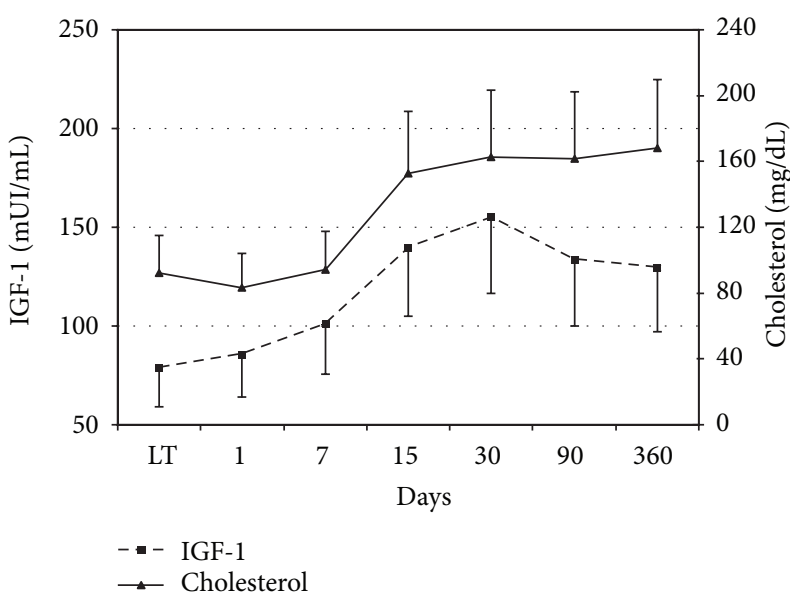

(a)

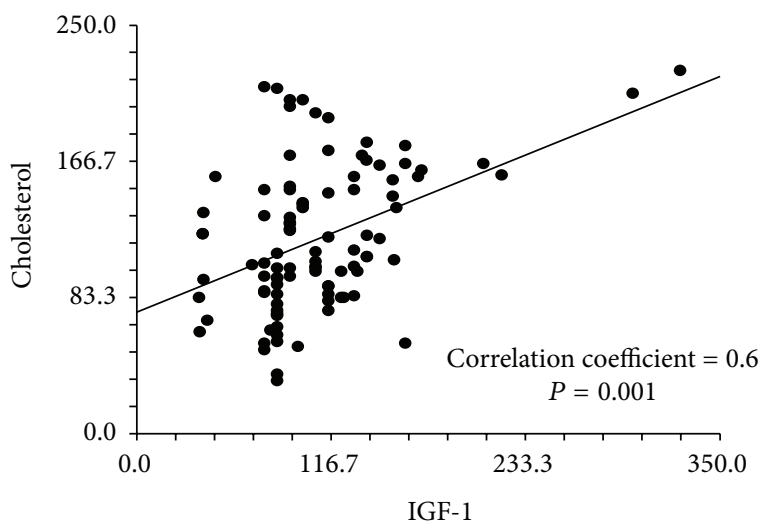

(b)

Figure 2: (a) Mean values of IGF-1 and cholesterol at liver transplantation (LT) and after transplant until day 360 are reported. IGF1 and cholesterol results are plotted according to left and right axis, respectively. (b) IGF-1 and cholesterol correlation plot is depicted together with correlation coefficient and $P$ value.

$(P<0.001)$ and IGF-1 mUI/mL $57 \pm 16$ versus $125 \pm 36$ in controls $(P<0.001)$. Liver transplantation determined a progressive normalization of the mean values of these two hormones. During the recovery phase after surgery IGF-1 and $\mathrm{GH}$ plasma levels were inversely correlated. However, this correlation was not strict (Pearson $r=-0.2 ; P=0.05$ ). Correlation analysis of GH and IGF-1 with other biochemical patient variables showed a correlation between IGF-1 and cholesterol levels (Figure 2).

3.3. Comparison of Serum Levels of GH and IGF-1 among Survivors and Nonsurvivors. GH and IGF-1 serum levels of surviving and nonsurviving patients were compared after LT (Table 2). Serum analysis was restricted to the first month after transplant as all nonsurvivors died within 90 days of surgery. Patients with a favourable outcome exhibited lower levels of GH at day 15 and higher levels of IGF-1 at days 15 and 30 after transplantation. These differences were statistically significant (Table 2). In order to identify cut-off values for
TABLE 2: Comparison of GH and IGF-1 serum levels in short-term (>3 months) survivors and nonsurvivors after liver transplantation.

\begin{tabular}{lccc}
\hline Days after LT & $\begin{array}{c}\text { Survivor } \\
(n=23)\end{array}$ & $\begin{array}{c}\text { Nonsurvivor } \\
(n=7)\end{array}$ & Statistics \\
\hline & & $\mathrm{GH}$ & \\
1 & $13.9 \pm 18$ & $13.6 \pm 10.6$ & n.s. \\
7 & $9.9 \pm 15.1$ & $11 \pm 8.2$ & n.s. \\
15 & $5.8 \pm 7.3$ & $18.8 \pm 27$ & $P=0.04$ \\
30 & $10.2 \pm 16.8$ & $20.1 \pm 33.6$ & n.s. \\
\hline & & IGF-1 & \\
1 & $85.2 \pm 23.7$ & $86.5 \pm 27.7$ & n.s. \\
7 & $100.9 \pm 35.7$ & $101.5 \pm 27.4$ & n.s. \\
15 & $162.8 \pm 105.6$ & $67.1 \pm 35$ & $P=0.04$ \\
30 & $175.4 \pm 94$ & $79 \pm 4.3$ & $P=0.03$ \\
\hline
\end{tabular}

Results are expressed as mean \pm standard deviation. n.s.: difference not statistically significant.

serum levels of GH (day 15) or IGF-1 (days 15-30) predictive of LT outcome, ROC curves were constructed with these two variables. For GH levels a cut-off value of $8.3 \mu \mathrm{UI} / \mathrm{mL}$ was identified but with a very limited sensitivity and specificity (70\% and 30\%, resp.; AUC $=0.4$ ). Conversely, analysis of IGF-1 data showed an unfavourable outcome in patients presenting serum levels $<90 \mathrm{mUI} / \mathrm{mL}$ at days 15 and 30 . This IGF-1 cut-off value showed a sensitivity of $86 \%$ and a specificity of $87 \%$ (AUC $92 \%$ ) at day 15 and a sensitivity of $86 \%$ and a specificity of $95 \%$ (AUC $96 \%$ ) at day 30 in predicting patient survival. Dot plot analysis (days 15 and 30) with the corresponding cut-off value is shown in Figure 3. Survival analysis was also conducted with the same IGF-1 cutoff value of $90 \mathrm{mUI} / \mathrm{mL}$ as illustrated in Figure 4 . Analysis showed a statistically significant increase in short-term (3 months) mortality in patients with IGF-1 serum levels $<90$ $\mathrm{mUI} / \mathrm{mL}$ between days 15 and 30 after liver transplant $(P<$ 0.001 Log Rank test).

\section{Discussion}

Short-term mortality after LT remains an open issue, with minor differences between Europe and United States [24, 25]. Data from the European Liver Transplant Registry (ELTR) show that, in a ten-year follow-up, approximately half of the patients transplanted die within the first six months [24]. Prompt identification of patients with poor short-term prognosis after LT may be useful in making clinical decisions that may possibly improve the outcome for these subjects. Unfortunately, the Model for End Stage Liver Disease (MELD) and Child-Pugh scores that are efficiently used for prognostic evaluation of liver diseases and priority of graft allocation lack a significant sensitivity and specificity in the assessment of short-term prognosis in LT patients when used in the pre- or early posttransplant periods [2628]. Simple evaluation of common biochemical indicators of liver function, such as albumin levels or coagulation function, is also not reliable, as supportive replacement therapy is frequently adopted in these patients. As a result, 


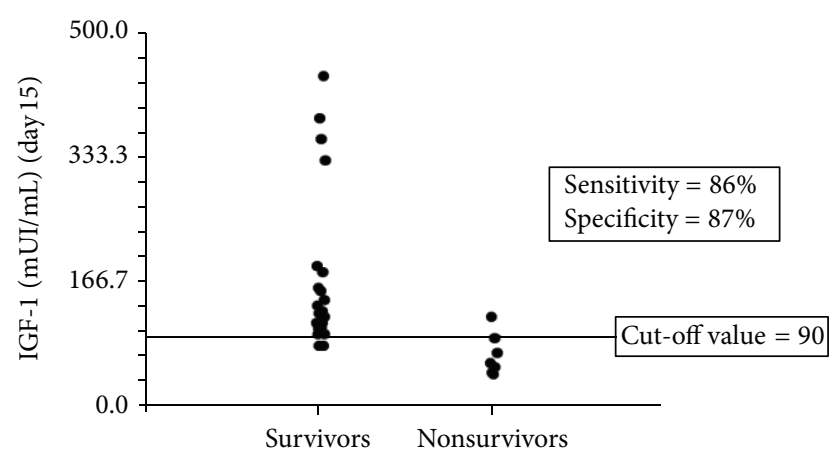

(a)

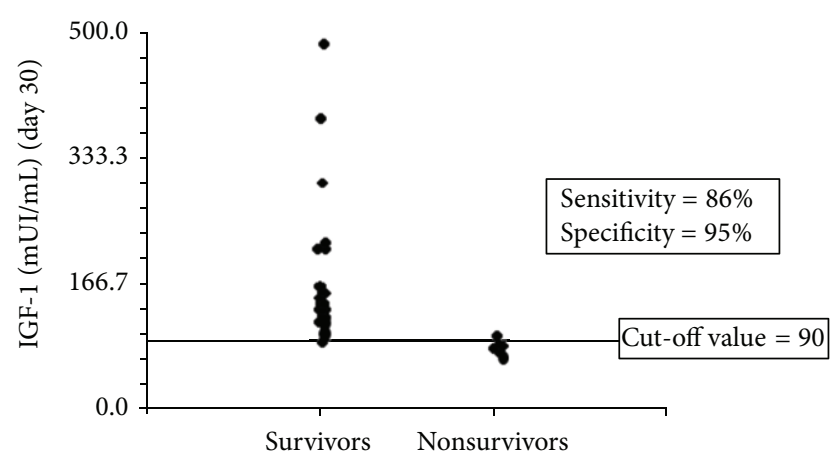

(b)

FIGURE 3: (a) Dot plot analysis of IGF-1 serum levels at day 15 after liver transplantation according to short-term ( $>3$ months) survival or nonsurvival. Sensitivity, specificity, and cut-off value are also reported. (b) Dot plot analysis of IGF-1 serum levels at day 30 after liver transplantation according to short-term ( $>3$ months) survival or nonsurvival. Sensitivity, specificity, and cut-off value are also reported.

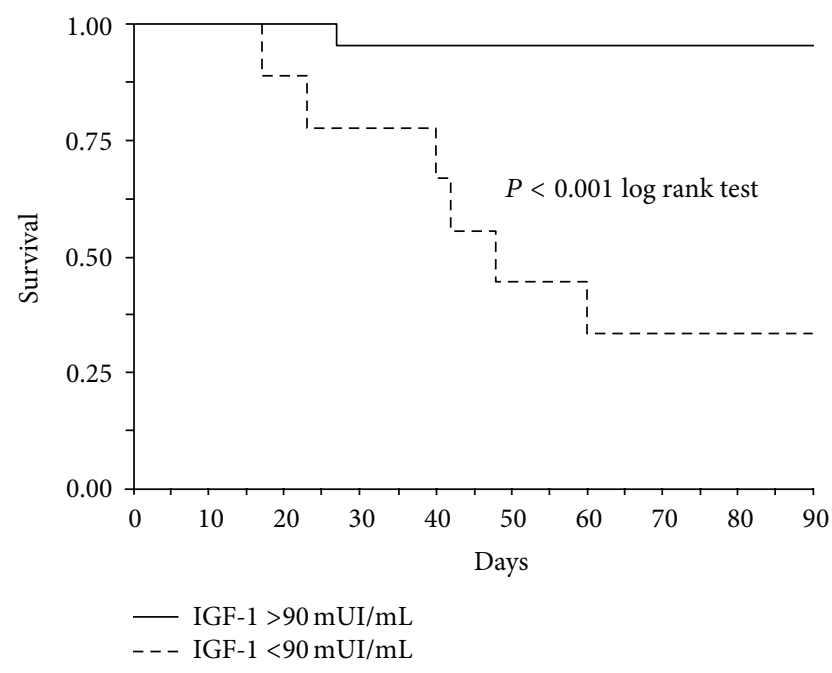

FIGURE 4: Short-term (3 months) survival analysis in patients presenting IGF-1 serum levels < (dotted line) or > (solid line) $90 \mathrm{mUI} / \mathrm{mL}$ between days 15 and 30 after liver transplant. Statistically significant difference $(P<0.001)$ by Log Rank test. scoring systems not designed to evaluate posttransplant liver function, such as the RIFLE (risk of renal dysfunction, injury to the kidney, failure of the kidney, loss of kidney function, and end stage kidney disease) tailored to assess the severity of acute renal failure or Sequential Organ Failure Assessment (SOFA) designed to predict general morbidity in the intensive care unit, have a better performance [28]. In the light of this background we decided to evaluate whether GH or IGF1 serum levels may be associated with graft recovery and patient survival after LT. IGF-1 is a hormone which is mainly produced by the hepatocytes. In this perspective the liver has a central endocrine role in the GH-IGF-1 axis [29]. IGF-1 deficiency has been well described in chronic liver diseases. The corresponding malnutrition status evidenced in cirrhosis has been suggested by some authors to be related to the limited production of IGF-1 by the diseased liver $[8,10,29]$. As regards LT a possible prognostic value of IGF-1 serum assessment after grafting has already been suggested. Bassanello and coauthors conducted a study on this issue in a limited number of patients [18]. Unfortunately, short-term mortality was not examined and the trend of IGF-1 serum levels was analysed only as a function of IPF of the graft. In this regard a statistically significant increase in IGF-1 was reported in IPF patients. We reassessed this issue in a study including a larger number of patients and with a longer follow-up. Our data firstly demonstrated a correlation between IGF-1 and cholesterol serum levels. The linear correlation between IGF-1 and cholesterol, both values showing a posttransplant progressive stepwise increase, suggests their relationship with graft functional recovery.

Given the variations of the GH/IGF-1 axis in the early phases after LT and the specific IGF-1 relationship with the graft restoring process, we evaluated the possible changes of these two hormones in short-term survivors and nonsurvivors after LT. Our data demonstrated that, already in the second week after surgery, liver related short-term survivors had decreased serum levels of IGF-1. The subsequent statistical analysis showed that patients with levels of IGF-1 persistently lower than $90 \mathrm{mUI} / \mathrm{mL}$, after the first week, had only a 13-5\% possibility of survival on the short-term. On the other hand, the same cut-off identified nonsurvivors in $86 \%$ of cases. The results were also confirmed by survival analysis. These data seem encouraging considering the scarcity of liver specific tests to apply after LT in the attempt to forecast shortterm mortality. Early retransplantation, even if controversial for ethical and economical reasons, may be the sole option to improve the outcome of these patients in selected cases [30, 31]. However, in the absence of valid prognostic parameters, this clinical strategy cannot be pursued without an acceptable indication such as primary nonfunction and hepatic artery thrombosis. Even though we understand that the encouraging data from this single centre, preliminary, retrospective study need to be confirmed in larger series, our results suggest a possible strategy to gather clinical information related to liver function restoration after LT and possibly evaluate shortterm survival. Whether exogenous therapeutic restoration of normal GH-IGF-1 levels may improve LT outcomes remains speculative at this stage. 


\section{Abbreviations}

(GH): Growth hormone

(IGF-1): Insulin like growth factor-1

(LT): Liver transplantation

(IPF): Initial poor function

(ROC): Receiver operating characteristic

(ALT): Alanine transaminase.

\section{Conflict of Interests}

The authors declare that there is no conflict of interests regarding the publication of this paper.

\section{Authors' Contribution}

All authors actively participated in the development of this study.

\section{Acknowledgment}

The authors express gratitude to Serena Rotunno for revision of the English text.

\section{References}

[1] R. G. Rosenfeld and V. Hwa, "The growth hormone cascade and its role in mammalian growth," Hormone Research, vol. 71, supplement 2, pp. 36-40, 2009.

[2] A. M. Lichanska and M. J. Waters, "How growth hormone controls growth, obesity and sexual dimorphism," Trends in Genetics, vol. 24, no. 1, pp. 41-47, 2008.

[3] D. E. Berryman, J. S. Christiansen, G. Johannsson, M. O. Thorner, and J. J. Kopchick, "Role of the GH/IGF-1 axis in lifespan and healthspan: lessons from animal models," Growth Hormone and IGF Research, vol. 18, no. 6, pp. 455-471, 2008.

[4] M. Rincon, E. Rudin, and N. Barzilai, "The insulin/IGF-1 signaling in mammals and its relevance to human longevity," Experimental Gerontology, vol. 40, no. 11, pp. 873-877, 2005.

[5] G. Mariño, A. P. Ugalde, A. F. Fernández et al., "Insulinlike growth factor 1 treatment extends longevity in a mouse model of human premature aging by restoring somatotroph axis function," Proceedings of the National Academy of Sciences of the USA, vol. 107, pp. 16268-16273, 2010.

[6] E. Shmueli, M. Stewart, K. G. M. M. Alberti, and C. O. Record, "Growth hormone, insulin-like growth factor-1 and insulin resistance in cirrhosis," Hepatology, vol. 19, no. 2, pp. 322-328, 1994.

[7] Y. Baruch, N. Assy, T. Amit et al., "Spontaneous pulsatility and pharmacokinetics of growth hormone in liver cirrhotic patients," Journal of Hepatology, vol. 29, no. 4, pp. 559-564, 1998.

[8] M. C. Blomsma, R. J. de Knegt, R. P. F. Dullaart, and P. L. M. Jansen, "Insulin-like growth factor-I in liver cirrhosis," Journal of Hepatology, vol. 27, no. 6, pp. 1133-1138, 1997.

[9] K. Bonefeld and S. Møller, "Insulin-like growth factor-I and the liver," Liver International, vol. 3, pp. 911-919, 2011.

[10] L. Caregaro, F. Alberino, P. Amodio et al., "Nutritional and prognostic significance of insulin-like growth factor 1 in patients with liver cirrhosis," Nutrition, vol. 13, no. 3, pp. 185-190, 1997.
[11] L. Caregaro, F. Alberino, P. Angeli, and A. Gatta, "Insulinlike growth factor 1 (IGF-1) in liver cirrhosis: a marker of hepatocellular dysfunction?" Journal of Hepatology, vol. 29, no. 2, article 342, 1998.

[12] N. Li, L. Zhou, B. Zhang et al., "Recombinant human growth hormone increases albumin and prolongs survival in patients with chronic liver failure: a pilot open, randomized, and controlled clinical trial," Digestive and Liver Disease, vol. 40, no. 7, pp. 554-559, 2008.

[13] M. Conchillo, R. J. De Knegt, M. Payeras et al., "Insulin-like growth factor I (IGF-I) replacement therapy increases albumin concentration in liver cirrhosis: results of a pilot randomized controlled clinical trial," Journal of Hepatology, vol. 43, no. 4, pp. 630-636, 2005.

[14] D. S. Schalch, M. Kalayoglu, J. D. Pirsch, H. Yang, M. Raslich, and S. Rajpal, "Serum insulin-like growth factors and their binding proteins in patients with hepatic failure and after liver transplantation," Metabolism: Clinical and Experimental, vol. 47, no. 2, pp. 200-206, 1998.

[15] M. M. Weber, C. J. Auernhammer, P. D. K. Lee, D. Engelhardt, and R. Zachoval, "Insulin-like growth factors and insulin-like growth factor binding proteins in adult patients with severe liver disease before and after orthotopic liver transplantation," Hormone Research, vol. 57, no. 3-4, pp. 105-112, 2002.

[16] D. Seehofer, T. Steinmueller, K.-J. Graef et al., "Pituitary function test and endocrine status in patient with cirrhosis of the liver before and after hepatic transplantation," Annals of Transplantation, vol. 7, no. 2, pp. 32-37, 2002.

[17] G. R. Castro, J. C. Coelho, M. B. Parolin, J. E. Matias, and A. C. de Freitas, "Insulin-like growth factor I correlates with MELD and returns to normal level after liver transplantation," Annals of Transplantation, vol. 18, pp. 57-62, 2013.

[18] M. Bassanello, E. F. de Palo, F. Lancerin et al., "Growth hormone/insulin-like factor 1 axis recovery after liver transplantation: a preliminary prospective study," Liver Transplantation, vol. 10, no. 5, pp. 692-698, 2004.

[19] L. Urbani, A. Campatelli, J. Romagnoli et al., "T-tube removal after liver transplantation: a new technique that reduces biliary complications," Transplantation, vol. 74, no. 3, pp. 410-413, 2002.

[20] L. Falasca, G. Tisone, G. Palmieri et al., "Protective role of tauroursodeoxycholate during harvesting and cold storage of human liver," Transplantation, vol. 71, no. 9, pp. 1268-1276, 2001.

[21] L. Baiocchi, M. Angelico, A. Petrolati et al., "Correlation between liver fibrosis and inflammation in patients transplanted for HCV liver disease," American Journal of Transplantation, vol. 8, no. 3, pp. 673-678, 2008.

[22] L. Baiocchi, F. De Leonardis, M. Delle Monache et al., "Plasma/erythrocyte ribavirin x100 ratio as an indicator of sustained virological response in HCV genotype 1 patients with early virological response," Antiviral Therapy, vol. 15, no. 4, pp. 633-639, 2010

[23] P. Cesaro, A. Kohn, L. Petruziello et al., "A survey on mortality from non-variceal upper gastrointestinal bleeding: is the emergency referral system adequate?" Digestive and Liver Disease, vol. 45, pp. 953-956, 2013.

[24] P. Dutkowski, O. De Rougemont, B. Müllhaupt, and P.-A. Clavien, "Current and future trends in liver transplantation in Europe," Gastroenterology, vol. 138, no. 3, pp. 802-809, 2010.

[25] C. L. Berg, D. E. Steffick, E. B. Edwards et al., "Liver and intestine transplantation in the United States 1998-2007," The American Journal of Transplantation, vol. 9, no. 4, pp. 907-931, 2009. 
[26] P. H. Hayashi, L. Forman, T. Steinberg et al., "Model for end-stage liver diseases score does not predict patient or graft survival in living donor liver transplant recipients," Liver Transplantation, vol. 9, no. 7, pp. 737-740, 2003.

[27] N. M. Desai, K. C. Mange, M. D. Crawford et al., "Predicting outcome after liver transplantation: utility of the model for endstage liver disease and a newly derived discrimination function," Transplantation, vol. 77, no. 1, pp. 99-106, 2004.

[28] C.-S. Wong, W.-C. Lee, C.-C. Jenq et al., "Scoring short-term mortality after liver transplantation," Liver Transplantation, vol. 16, no. 2, pp. 138-146, 2010.

[29] E. F. De Palo, M. Bassanello, F. Lancerin et al., "GH/IGF system, cirrhosis and liver transplantation," Clinica Chimica Acta, vol. 310, no. 1, pp. 31-37, 2001.

[30] R. Pfitzmann, B. Benscheidt, J. M. Langrehr, G. Schumacher, R. Neuhaus, and P. Neuhaus, "Trends and experiences in liver retransplantation over 15 years," Liver Transplantation, vol. 13, no. 2, pp. 248-257, 2007.

[31] R. M. Ghobrial, "Changing faces of liver retransplantation: it is all about selection," Liver Transplantation, vol. 13, no. 2, pp. 188$189,2007$. 


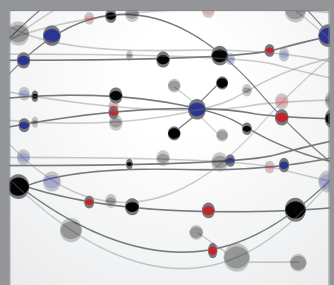

The Scientific World Journal
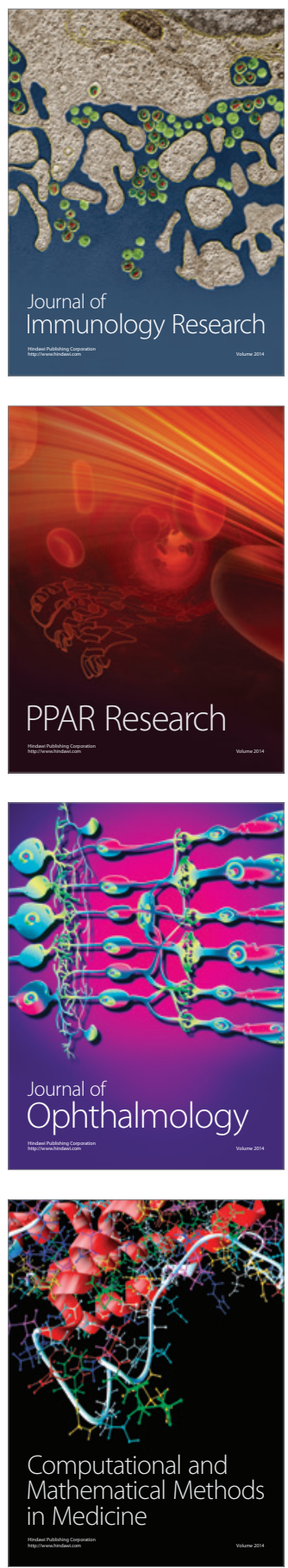

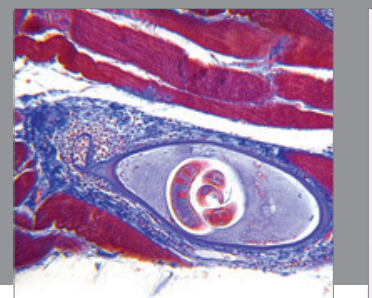

Gastroenterology

Research and Practice
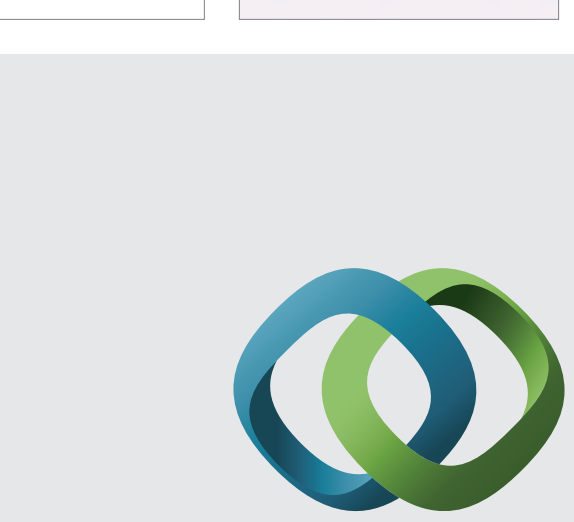

\section{Hindawi}

Submit your manuscripts at

http://www.hindawi.com
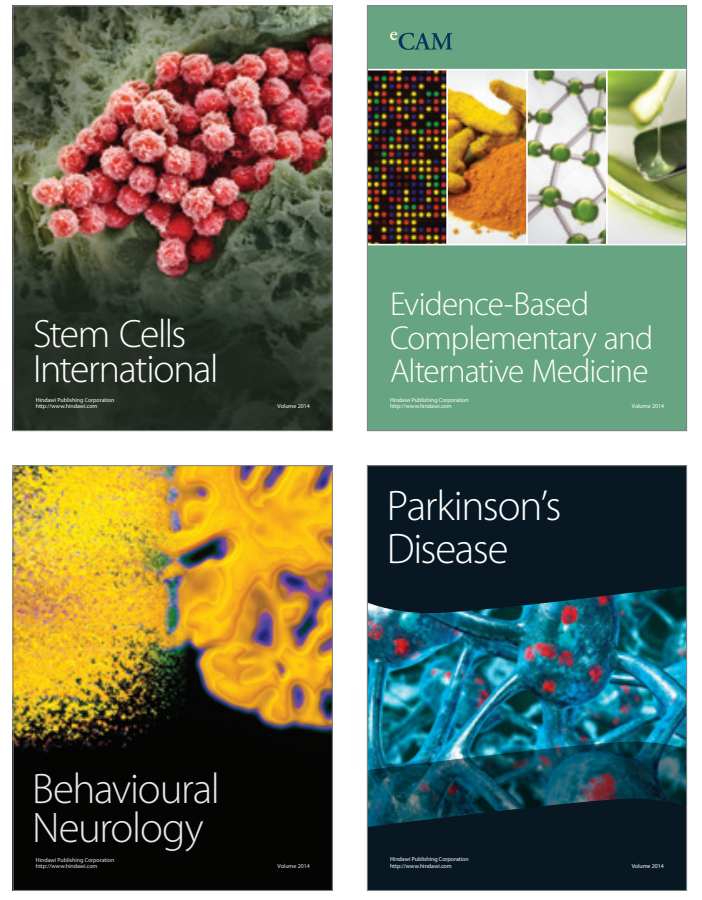
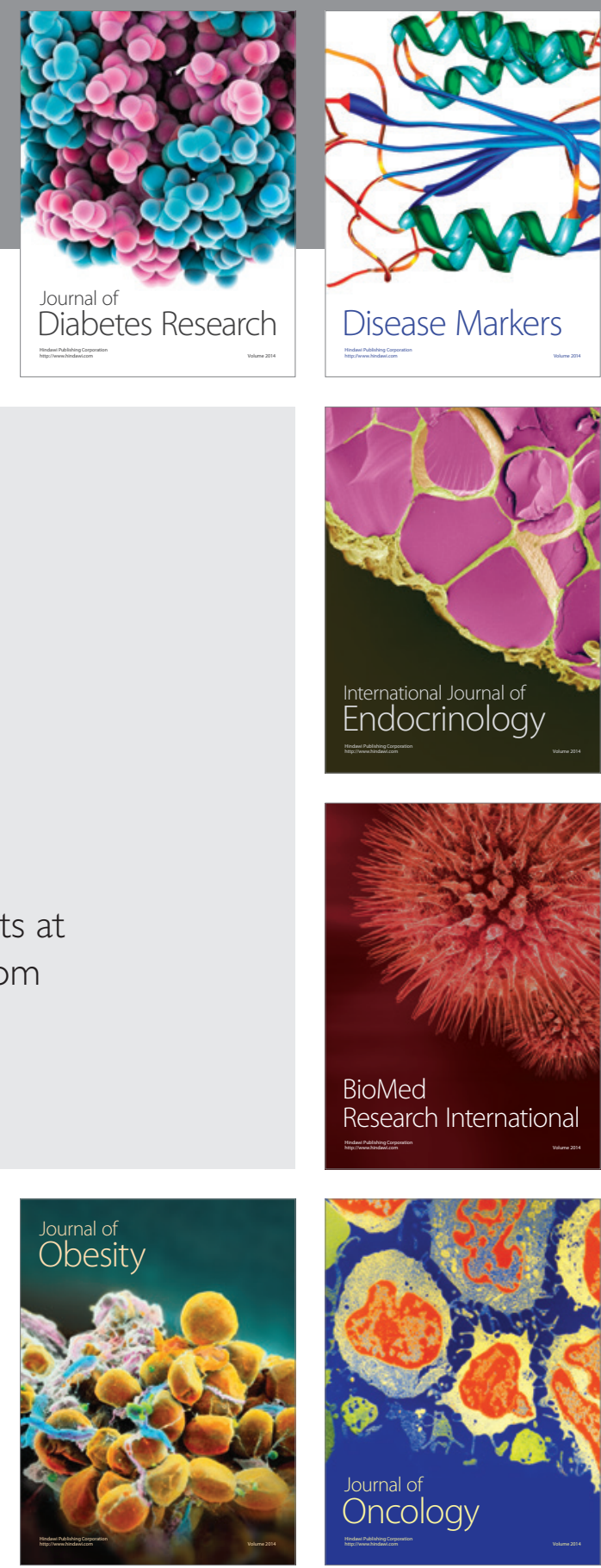

Disease Markers
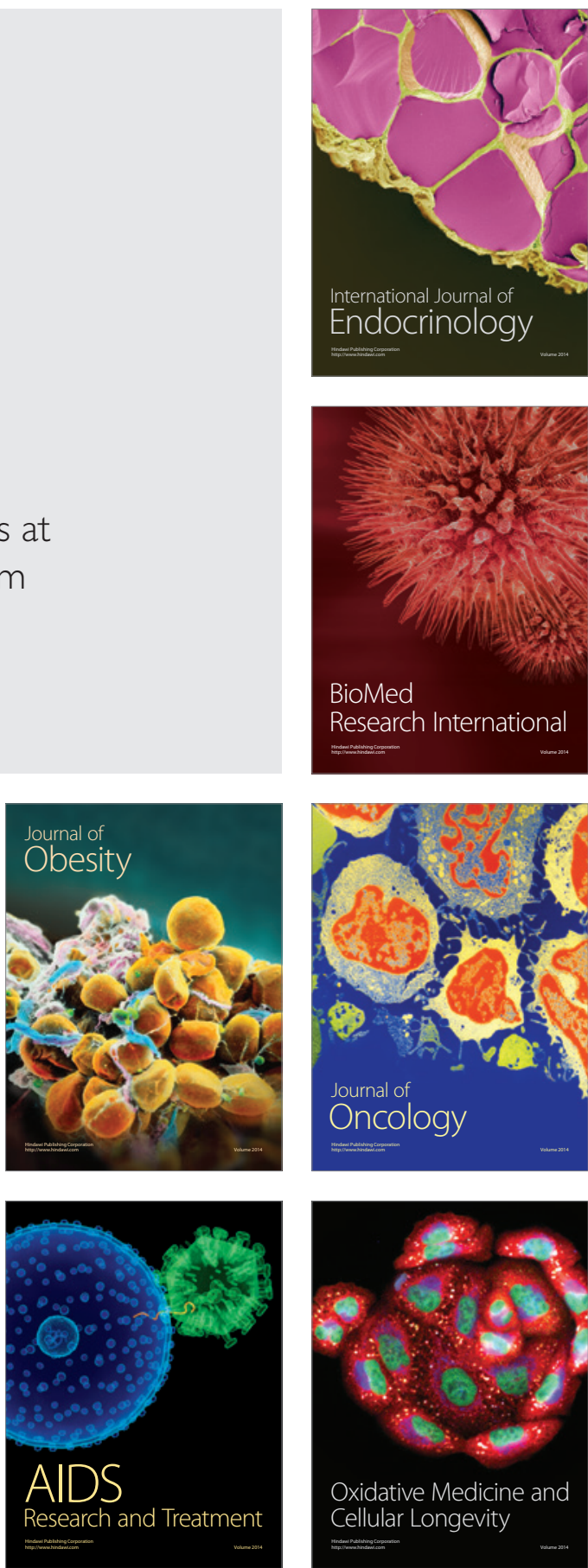